\title{
Geometrical Transformation Approximation for 2D/3D Intensity-Based Registration of Portal Images and CT Scan
}

\author{
David Sarrut ${ }^{1}$ and Sébastien Clippe ${ }^{2}$ \\ 1 ERIC laboratory \\ Université Lumière Lyon 2 \\ 5 Av. Pierre Mendès-France - 69676 Bron - FRANCE \\ dsarrut@univ-lyon2.fr \\ 2 Centre de Lutte Contre le Cancer Léon Bérard \\ 28, rue Laënnec - 69008 Lyon - FRANCE \\ sclippe@univ-lyon2.fr
}

\begin{abstract}
Conformal radiotherapy treatments need accurate patient positioning in order to spare normal tissues. Patient pose can be evaluated by registering portal images (PI) with Digitally Reconstructed Radiographs (DRR). Several methods involve segmentation which is known to be a difficult task for noisy PI. In this paper, we study another approach by using a fully $3 \mathrm{D}$ intensity-based registration method, without segmentation. Our approach uses the correlation ratio as similarity measure and replace DRR generation with a treatment on pre-computed DRR. A specific geometrical transformation is applied to approximate a given projection by the composition of out-of-plane rotations and inplane transformation. Some preliminary experiments on both simulated and real portal images, lead to good results (RMS error lower than 2 $\mathrm{mm})$.
\end{abstract}

\section{Medical Context}

Introduction This work is done in collaboration with physicians from Lyon's Léon Bérard Institute (France) and concerns cancer treatment by conformal radiotherapy. The goal of radiotherapy is to accurately deliver a curative dose of X-rays (produced by linear accelerators) to the tumor while sparing surrounding normal tissues. With help of a computed tomography (CT) scan, physicians create a Radiotherapy Treatment Planning (RTP) which plans irradiation sessions, with one irradiation per day during several weeks. During each session, the patient must be in the same position as he was during CT acquisition.

However, it is a very difficult task to exactly position the patient in the same position each day and studies have shown that setup inaccuracies could lead to poorer local control or survival [1]. Numerous studies have evaluated displacements (see [2, 3]), they relate a mean setup error between $5.5 \mathrm{~mm}$ and $8 \mathrm{~mm}$ (the maximum could reach $18 \mathrm{~mm}$ ). Even in recent series, displacements still remain important: $22 \%$ of displacements are between 5 and $10 \mathrm{~mm}$ [2] 
and $57 \%$ greater than $4 \mathrm{~mm}$ [3], despite the use of immobilization devices (such as polyurethan foam cast or thermoplastic mask). Such systems generally reduce setup errors but without eliminating all errors 4. In order to control patient pose, a solution is to use images from EPID (Electronic Portal Imaging Devices) 5]. Portal Images (PI) are 2D projection images and are acquired with the irradiation device. By visual inspection, physicians have the possibility to roughly correct the position of the patient, but it is both inaccurate and timeconsuming. Moreover, as conformal radiotherapy uses smaller margins around the target volume, high precision becomes more and more needed to be sure that no target is missed, with the risk of local recurrence.

We propose in this study an automatic method for setup errors evaluation. The next section presents related work, section 2 described the geometrical part of the registration and the intensity-based similarity measures. Experiments and results are in the section 3 .

Related Works Several image registration methods have been used to compare control images (obtained from EPID or other modalities such as ultrasound images [6] or radiographic film [7]) with a reference image (CT scan). Most of the studies on this subject use a segmentation procedure: some features (bony structures) are extracted from the images and registered. Bijhold et al. [7] use a manual segmentation of bony outlines visible in both portal (a film) and reference images. Other methods [8, 3] use anatomical landmarks: several homologous points are determined in both images and then matched. Marker-based methods have also be proposed [9] 10]: they consist in the implantation of radio-opaque markers inside the body of the patient. However, the markers have to be fixed in the tumor volume, which is an important restriction for implantation. Fully 3D method was proposed in [11], and was based on the registration of a 3D surface extracted from the CT scan with several image contours, segmented from the PI. Numerous methods use digitally reconstructed radiographs (DRR). DRR are 2D projection images computed by a specific volume-rendering (pinhole projection model) from the CT-scan. Gilhuijs et al. [12] developed a 3D method with partial (segmented) DRR. However, the segmentation is a difficult task and often fails 13. because PI have very low contrast (due to the high energy, 5-20 Megavolt). In order to avoid or help segmentation, some authors use intensity-based methods, based on a similarity measure computed with the value of (potentially) all the pixels (see section 2.3). Few works about 3D image registration using such techniques in this context have been published [14, 15, 16, 17.

\section{Intensity-Based 2D/3D Registration}

\subsection{Overview of the Method}

In this work, we focus on rigid transformation, denoted by $T$ (3 translation and 3 rotation parameters). The CT scan is denoted by $\mathcal{V}$. In-plane (2D) registration of a single DRR with a single PI is known to be inaccurate in case of out-of-plane 
rotations or large translations [7, 18, 13]. Hence, we use several PI and several DRR. In practical situation, due to the limited amount of radiation received by the patient, two $(n=2) \mathrm{PI}$ are acquired from orthogonal viewpoints. The $i^{\text {th }} \mathrm{PI}$ is denoted by $\mathcal{I}_{i}$, and the corresponding projection matrices (obtained with a calibration procedure) are denoted by $Q_{i}$. Given a similarity measure $\mathcal{S}$, and $\mathcal{I}$ the vector of $n$ PI's, the main $3 \mathrm{D}$ optimization procedure is:

$$
\widehat{T}=\arg _{T} \max \mathcal{S}(\boldsymbol{Q T}(\mathcal{V}), \mathcal{I})
$$

In eq.(1), $Q \boldsymbol{T}(\mathcal{V})$ denotes a vector of $n$ DRR, according to the $n$ projection $Q_{i}$ and the patient displacement $T$. Each iteration of eq.(1) requires the (on-line) generation of $n$ DRR. However, this is a too long process to be tractable. Several authors have study ways to speedup DRR generation [19, 12, 16, at the cost of DRR's quality (and thus the quality of the similarity criterion). We choose to study another solution which is to generate a set of DRR before the registration stage, when there is no time limitation.

\subsection{Geometrical Transformation Approximation for DRR Generation}

During an iteration of eq. (1), a DRR must be generated from a given projection $Q T$ (subscript $i$ is omitted for clarity). It is obviously impossible to pre-generate DRR from all the possible orientations. Moreover, the space $P$ of projections, even bounded to the space of plausible projections (e.g. rotations lower than 10 degrees, translations lower than $2 \mathrm{~cm}$ ) has 6 dimensions, and it is difficult to efficiently sample this space. Our main idea is to reduce the dimensionality of $P$ by only considering out-of-plane rotations (2 parameters) and using inplane (2D) transformations to retrieve ideal projection $Q T$. This decomposition assumes that the $3 \mathrm{D}$ displacements which make in-plane registration fail, are due to out-of-plane rotations. Obviously, this is not theoretically true because the projection model is a pinhole one. However, the distance between the camera and the patient is much more larger than the patient displacement amplitude, and our experiments show that this approximation is sufficient for our purpose. We are thus looking for a decomposition, described by out-of-plane rotations (apply before projection by $Q$ ) and by in-plane transformation (after $Q$ ), which is the closest to the projection of a given position $Q T$.

We denote by $O z$ the projection direction. We decompose a $3 \mathrm{D}$ rotation into out-of-plane and in-plane rotations. In-plane rotation is around axe $O z$. We denote by $R_{\alpha, \Delta}$ the out-of-plane rotation of angle $\alpha$ around the axe $\Delta$. Axe $\Delta$ goes through the iso-center $O$, is orthogonal to the projection direction $O z$, and $\Delta$ is parameterized with a single parameter, the angle $\beta$ according to the $O x$ axe (see figure 1). Then, the objective projection $Q T$ is replaced by $L Q R_{\alpha, \Delta}$, with $L$ an in-plane transformation. In order to find $\alpha, \beta$ and $L$ for a given $T$, we perform a least square optimization, see eq.(2).

$$
\left.\begin{array}{l}
L_{T} \\
R_{T}
\end{array}\right\}=\arg _{L, \alpha, \Delta} \min \sum_{\boldsymbol{x} \in H}\left(Q T(\boldsymbol{x})-L Q R_{\alpha, \Delta}(\boldsymbol{x})\right)^{2}
$$




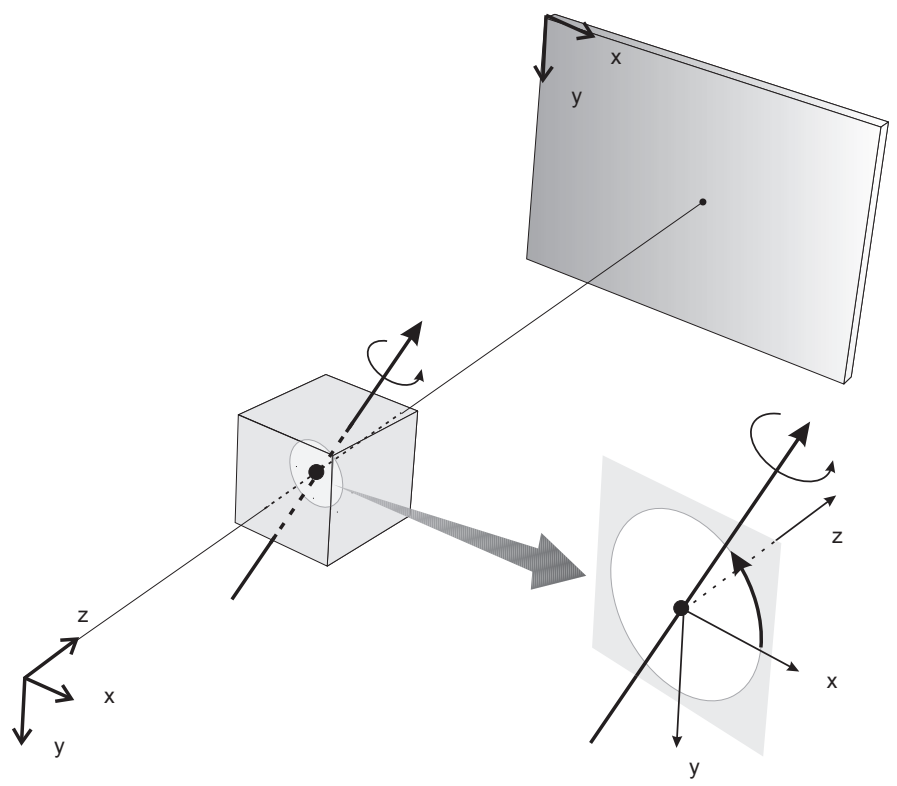

Fig. 1. Out-of-plane rotation according to a projection direction $O z$, parameterized with angle $\alpha$ around axe $\Delta$ in the $O x O y$ plane.

In eq.(2), $L$ can have 3 (2 translations and a rotation) or 4 parameters (with a scaling factor). The set $H$ is composed of random points $x \in \mathbb{R}^{3}$. This optimization is performed with the Powell-Brent method described in [20]. The subspace of $P$, described with the two parameters $\alpha$ and $\beta$, can now be sampled. By bounding such space to plausible rotations and by choosing a sampling rate (for example 0.5 degrees), it is possible to generate a set DRR before patient treatment. In conclusion, we replace the expensive DRR generation required at each iteration of eq. (11) by a two-pass approach: optimization eq.(2) and application of the in-plane transformation $L_{T}$ on the pre-computed DRR given by projection $R_{T}$.

\subsection{Intensity-Based Similarity Measures}

Joint Histograms. The relative position of two images is evaluated with an intensity-based similarity measure which requires no segmentation. Measures, such as correlation coefficient [21], mutual information [22, 23] or correlation ratio [24, are all based on joint histograms (even if we do not need to explicitly compute it). A joint histogram, denoted by $H_{L}$, is a $2 \mathrm{D}$ histogram computed according to a transformation $L$ between the two images. Quantities $H_{L}(i, j)=$ $n_{i j}$ are computed by summing for each couple of intensities $(i, j)$, the number of co-occurrent pixels. Probabilities $p_{i j}\left(p_{i}=\sum_{j} p_{i j}, p_{j}=\sum_{i} p_{i j}\right)$ must then be estimated from $n_{i j}$. Most authors used a frequential estimation $\left(p_{i j}=\frac{n_{i j}}{n}\right.$, 
with $n$ the number of points in the overlapping part of the images). As advocated in [23], we use Parzen windowing to estimate $p_{i j}$ with a Gaussian density function (probabilities are locally averaged).

A criterion $\mathcal{S}$ measures some type of dependence between the two distributions: according to the assumptions made on the type of the dependence (e.g. linear, functional), on the type of variable (numerical or categorical), and by using different diversity measures (variance, entropy), several measures can be defined 25 .

Study of Several Similarity Measures. We studied four measures. The correlation coefficient (CC 26, 21]), measures the strength of a linear relation between intensity distributions. $\chi^{2}$ measures a distance to the independence case (where $p_{i j}=p_{i} p_{j} \forall i, j$ ). Mutual information (MI 22, 23]) uses relative Shannon's entropy to also compute a distance to the independence case. MI is maximal if a functional dependence exists between the intensities. The correlation ratio (CR [24) also assumes a functional relation but measures its strength by the way of a proportional reduction of variance. Following equations express the three measures (images are denoted $I$ and $J$, mean is $m_{I}=\sum_{i} i p_{i}$, variance is $\sigma_{I}^{2}=\sum_{i}\left(i-m_{I}\right)^{2} p_{i}$, and the conditional variance $\left.\sigma_{I \mid j}^{2}=\frac{1}{p_{j}} \sum_{i}\left(i-m_{I \mid j}\right)^{2} p_{i j}\right)$ :

$$
\begin{aligned}
C C(I, J) & =\sum_{i j} \frac{\left(i-m_{I}\right)\left(j-m_{J}\right)}{\sigma_{I} \sigma_{J}} p_{i j} & \chi^{2}(I, J) & =\sum_{i j} \frac{\left(p_{i j}-p_{i} p_{j}\right)^{2}}{p_{i} p_{j}} \\
M I(I, J) & =\sum_{i j} p_{i j} \log \frac{p_{i j}}{p_{i} p_{j}} & C R(I \mid J) & =1-\frac{1}{\sigma_{I}^{2}} \sum_{j} p_{j} \sigma_{I \mid j}^{2}
\end{aligned}
$$

Interpolation is performed by partial volume [22]. CR is not a symmetric criterion (whereas the others are) and an image $(I)$ must be chosen to estimate the other $(J)$ : the optimization eq. (1) compares the same PI with several DRR and $\mathrm{CR}$ is normalized according to $I$, so we decided to choose $I=D R R$ and $J=P I$. We do not discuss further the properties of such measures, interested reader could see [22, 23, 24, 25].

In eq.(1), $\mathcal{S}$ must quantify similarity between a vector of images couples. A solution can be to perform a linear combination of the similarity values between each couples $\sum_{i}^{n} \alpha_{i} \mathcal{S}\left(D R R_{i}, \mathcal{I}_{i}\right)$. However, it is not clear how to determine the weights $\alpha_{i}$. So, we propose the following original solution: for each images couples, we update the same joint histogram. Then, a similarity measure (whatever it is) can be computed from the unique histogram. Moreover, this force the criterion to measure the same type of dependence for all the images couples.

\section{Experiments}

Materiel and Method A CT-scan of an anthropomorphic phantom was acquired (88 slices, thickness $3 \mathrm{~mm}, 512^{2}$ pixels of size $0.87^{2} \mathrm{~mm}^{2}$ ). The artificial object was positioned on the irradiation table according to several random positions. For each position, two orthogonal PI were acquired (see figure 2). Size of 
irradiation field corresponds to realistic treatment (about $15^{2} \mathrm{~cm}$ at the isocenter, $280^{2}$ pixels). We performed two sets of experiments: with simulated data and with real PI. Simulated PI are generated from DRR computed from known displacement. Noisy aspect is simulated by smoothing the DRR and adding large Gaussian noise. Quality of such images was assessed by physician (see figure 2).

Parameters of the initial projection matrices $Q_{i}$ are obtained from a calibration procedure between CT and accelerator coordinate systems. For each position (with generated or real PI), we performed our method in order to obtain an estimation of the position. The set of pre-computed DRR is the following: outof-plane rotations were taken between $-7^{\circ}$ and $+7^{\circ}$ with a sample step $k=0.5^{\circ}$, leading to $30 \times 30=900$ images. Each estimation was performed with the same set of parameters, the starting point of the optimization was randomly chosen.
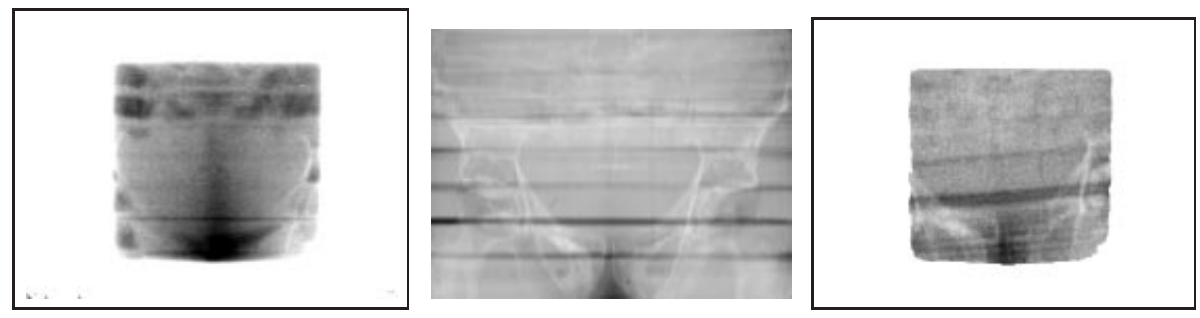

Fig. 2. Portal image, DRR and simulated PI.

Results For each position $i$ we compute the RMS (Root Mean Square) error, denoted by $\varepsilon_{i}$, between the estimation found by our method and the reference position. The RMS error is the average distance (in millimeter) between points transformed by the two compared transformations. We used 1000 points spread inside a cube of $15^{3} \mathrm{~cm}^{3}$ centered at the target point (the tumor) in order to obtain a realistic error; a RMS error of $x \mathrm{~mm}$ means that, in average, each point is $x \mathrm{~mm}$ away from the desired position. $\varepsilon_{r}$ denotes the RMS error of the displacement to be retrieve. Table 1 summarizes results on 100 positions from simulated images. It depicts mean, median and percentage of estimations which improve the initial position $\left(\varepsilon_{r}>\varepsilon_{i}\right)$. Table 2 summarizes results of tests on 7 real couples of PI. For each position, it depicts the initial error $\varepsilon_{r}$ and final error $\varepsilon_{i}$, obtained with different measures. Last lines depict mean and median RMS error. Estimations with RMS lower than $3 \mathrm{~mm}$ are displayed in grey boxes.

In the first experiment, the four measures are significantly different (using mixed model two ways ANOVA $\left.]^{1}\right), p<10^{-6}$. Using least significant difference test, neither CR and MI, nor CC and $\chi^{2}$ are significantly different (0.01 level). But, these two groups are significantly different. For CR and MI, RMS error

\footnotetext{
${ }^{1}$ Analyze of Variance
} 


\begin{tabular}{l|c|cccc} 
& $\varepsilon_{r}$ & CC & $\chi^{2}$ & MI & CR \\
\hline Mean & 10.07 & 3.78 & 4.63 & 2.10 & 2.75 \\
Median & 10.24 & 2.83 & 4.39 & 0.85 & 1.20 \\
$\varepsilon_{r}>\varepsilon$ & - & $92 \%$ & $94 \%$ & $99 \%$ & $99 \%$
\end{tabular}

Table 1. First experiment on simulated PI (100 positions): RMS error according to each similarity measure: mean, median, percentage of improvement.

\begin{tabular}{l|c|cccc} 
Positions & $\varepsilon_{r}$ & CC & $\chi^{2}$ & MI & CR \\
\hline 1 & 6.00 & 2.41 & 2.78 & 1.47 & 1.43 \\
2 & 6.00 & 9.79 & 12.83 & 21.00 & 2.00 \\
3 & 10.00 & 2.54 & 15.99 & 2.88 & 2.46 \\
4 & 7.00 & 2.52 & 1.18 & 2.68 & 1.29 \\
5 & 3.21 & 3.62 & 7.27 & 5.34 & 1.95 \\
6 & 5.29 & 5.38 & 3.36 & 5.82 & 4.33 \\
7 & 9.18 & 18.64 & 4.49 & 5.16 & 2.51 \\
\hline Mean & 5.83 & 5.61 & 5.99 & 5.54 & 1.99 \\
Median & 5.34 & 2.65 & 2.73 & 2.75 & 1.62
\end{tabular}

Table 2. Second experiment on real PI: initial RMS error $\left(\varepsilon_{r}\right)$ and final estimation error according to each similarity measure. Last two lines depict mean and median. Grey boxes emphasize RMS lower than $3 \mathrm{~mm}$.

decreases from $10 \mathrm{~mm}$ to $1.2 / 0.85 \mathrm{~mm}(\mathrm{CR} / \mathrm{MI})$. The second experiment shows weak results with $\mathrm{CC}$ and $\chi^{2}$, but also with MI. However, estimations with $\mathrm{CR}$ manage to recover patient pose in almost every cases (only one RMS is greater than 3) and always improve incorrect pose.

\section{Conclusion}

We have presented in this paper an original method for patient pose estimation using 2D portal images and 3D CT scan. The method is fully 3D, avoids segmentation, uses several PI, uses pre-generated DRR and is an intensity-based registration procedure. In the experimental tests, we obtain good position estimations both for simulated and real PI (median RMS is about $1.2 \mathrm{~mm}$ with simulated images and $1.6 \mathrm{~mm}$ for real PI). Our approach can be used with any number of PI (thanks to the unique JH, updated for each couple of images) and with any types of PI (by use of different similarity measures based on JH, such as $\mathrm{MI}$ or CR). The overall procedure is very fast, less than 3-4 minutes to complete on a common workstation (Ultra Sparc 5, $333 \mathrm{Mhz}$ ). The presented method is fully automatic and does not require any user intervention. The sampling of the set of pre-computed DRR plays an important role in the accuracy of the estimation. Experimental results show that $0.5^{\circ}$ is a good tradeoff between precision and volume storage. 
Further works are ongoing to improve the optimization procedure, and experiments on larger set of images are planned. The presented method is only valid for rigid body transformation, but we plan to study non-rigid deformations and organs displacements with the same principles (DRR generation and 2D transformations).

\section{Acknowledgments}

This work is supported by the Région Rhône-Alpes under the grant AdéMo ("Modélisation et suivi spatio-temporel pour le diagnostique et la thérapie").

\section{References}

[1] C. Carrie, S. Hoffstetter, and F. Gomez et al. Impact of targeting deviations on outcome in medulloblastoma: study of the french society of pediatric oncology (SFOP). Int. J. Radiat. Oncol. Biol. Phys., 45(2):435-9, September 1999.

[2] U. Mock, K. Dieckmann, U. Wolff, T.H. Knocke, and R. Potter. Portal imaging based definition of the planning target volume during pelvic irradiation for gynecological malignancies. Int. J. Radiat. Oncol. Biol. Phys., 45(1):227-232, 1999.

[3] J.C. Stroom, M.J. Olofsen van Acht, and S. Quint et al. On-line set-up corrections during radiotherapy of patients with gynecologic tumors. Int. J. Radiat. Oncol. Biol. Phys., 46(2):499-506, 2000.

[4] C.M. Nutting, V.S. Khoo, V. Walker, H. McNair, C. Beardmore, A. Norman, and D.P. Dearnaley. A randomized study of the use of a customized immobilization system in the treatment of prostate cancer with conformal radiotherapy. Radiother. Oncol., 54(1):1-9, 2000.

[5] P. Munro. Portal imaging technology: past, present, and future. Sem. Radiat. Oncol., 5(2):115-133, 1995.

[6] G. Ionescu, S. Lavalle, and J. Demongeot. Automated registration of ultrasound with ct images: Application to computer assisted prostate radiotherapy and orthopedics. In MICCAI'99, volume 1679 of $L N C S$, pages 768-777, Cambridge, England, September 1999.

[7] J. Bijhold, M. van Herk, R. Vijlbrief, and J.V. Lebesque. Fast evaluation of patient set-up during radiotherapy by aligning features in portal and simulator images. Phys. Med. Biol., 36(12):1665-79, 1991.

[8] J. Van de Steene, F. Van den Heuvel, and A. Bel et al. Electronic portal imaging with on-line correction of setup error in thoracic irradiation: clinical evaluation. Int. J. Radiat. Oncol. Biol. Phys., 40(4):967-976, 1998.

[9] K.L. Lam, R.K. Ten Haken, McShan DL, and Thornton AF Jr. Automated determination of patient setup errors in radiation therapy using spherical radioopaque markers. Med Phys, 20(4):1145-52, 1993.

[10] K.P. Gall, L.J. Verhey, and M. Wagner. Computer-assisted positioning of radiotherapy patients using implanted radiopaque fiducials. Med. Phys., 20(4):1153-9, 1993.

[11] S. Lavallée and R. Szeliski. Recovering the Position and Orientation of Free-Form Objects from Image Contours Using 3D Distance Maps. IEEE Transactions on Pattern Analysis and Machine Intelligence, 17(4):378-390, April 1995. 
[12] K.G. Gilhuijs, K. Drukker, A. Touw, P.J. van de Ven, and M. van Herk. Interactive three dimensional inspection of patient setup in radiation therapy using digital portal images and computed tomography data. Int. J. Radiat. Oncol. Biol. Phys., 34(4):873-85, 1996.

[13] P. Remeijer, E. Geerlof, L. Ploeger, K. Gilhuijs, M. van Herk, and J.V. Lebesque. $3-\mathrm{D}$ portal image analysis in clinical practice: an evaluation of 2-D and 3-D analysis techniques as applied to 30 prostate cancer patients. Int. J. Radiat. Oncol. Biol. Phys., 46(5):1281-90, 2000.

[14] D. Plattard, G. Champleboux, P. Vassal, J. Troccaz, and M. Bolla. EPID for patient positioning in radiotherapy: calibration and image matching in the entroPID system. In H.U. Lemke, editor, CARS'99, pages 265-9. Elsevier science, 1999.

[15] R. Bansal, L. Staib, Z. Chen, A. Rangarajan, J. Knisely, R. Nath R, and J. Duncan. A minimax entropy registration framework for patient setup verification in radiotherapy. Computer Aided Surgery, 4(6):287-304, 1999.

[16] R. Goecke, J. Weese, and H. Schumann. Fast volume rendering methods for voxelbased $2 \mathrm{~d} / 3 \mathrm{~d}$ registration - a comparative study. In International Workshop on Biomedical Image Registration, pages 89-102, Bled, Slovenia, August 1999.

[17] D. Sarrut and S. Clippe. Patient positioning in radiotherapy by registration of 2D portal to 3D CT images by a contend-based research with similarity measures. In Computer Assisted Radiology and Surgery, pages p 707-712, San Fransisco, USA, June 2000. Elsevier Science.

[18] J. Hanley, G.S. Mageras, J. Sun, and G.J. Kutcher. The effects of out-of-plane rotations on two dimensional portal image registration in conformal radiotherapy of the prostate. Int. J. Radiat. Oncol. Biol. Phys., 33(5):1331-1343, 1995.

[19] G.P. Penney, J. Weese, J.A. Little, P. Desmedt, D.L.G. Hill, and D.J. Hawkes. A comparison of similarity measures for use in 2D-3D medical image registration. IEEE Transaction on Medical Imaging, 17:586-595, 1998.

[20] W.H. Press, B.P. Flannery, S.A. Teukolsky, and W.T. Vetterling. Numerical Recipes in C: The Art of Scientific Computing. Cambridge University Press, second edition, 1992.

[21] D.H. Hristov and B.G. Fallone. A grey-level image alignment algorithm for registration of portal images and digitally reconstructed radiographs. Med Phys, 23(1):75-84, 1996.

[22] F. Maes, A. Collignon, D. Vandermeulen, G. Marchal, and P. Suetens. Multimodality Image Registration by Maximization of Mutual Information. IEEE Transaction On Medical Imaging, 16(2):187-198, April 1997.

[23] W.M. Wells, P.A. Viola, H. Atsumi, S. Nakajima, and R. Kikinis. Multi-Modal Volume Registration by Maximization of Mutual Information. Medical Image Analysis, 1(1):35-51, 1996.

[24] A. Roche, G. Malandain, X. Pennec, and N. Ayache. The Correlation Ratio as a New Similarity Measure for Multimodal Image Registration. In MICCAI'98, pages 1115-1124, Cambridge Massachusetts (USA), October 1998.

[25] D. Sarrut. Recalage multimodal et plate-forme d'imagerie médicale à accès distant. $\mathrm{PhD}$ thesis, Université Lumière Lyon 2, January 2000. In french.

[26] L. Dong and A.L. Boyer. An image correlation procedure for digitally reconstructed radiographs and electronic portal images. Int. J. Radiat. Oncol. Biol. Phys., 33(5):1053-60, 1995. 